\title{
Axial force coefficient of APFSDS projectile
}

\author{
Ammar Trakic ${ }^{1}$ \\ ${ }^{1}$ Defense Technologies Department, Mechanical Engineering faculty, University of Sarajevo; Bosnia and Herzegovina
}

*ammar.trakic@hotmail.com

(C) The Author

2020.

Published by

ARDA.

\begin{abstract}
Armor-piercing ammunition is primarily used to combat against heavy armored targets (tanks), but targets can be light armored vehicles, aircraft, warehouse, structures, etc. It has been shown that the most effective type of anti-tank ammunition in the world is the APFSDS ammunition (Armor Piercing Fin Stabilized Discarding Sabot). The APFSDS projectile flies to the target and with his kinetic energy acts on the target, that is, penetrates through armor and disables the tank and his crew. Since the projectile destroys target with his kinetic energy, then it is necessary for the projectile to have the high impact velocity.

The decrease in the velocity of a projectile, during flight, is mainly influenced by aerodynamic forces. The most dominant is the axial force due to the laid trajectory of the projectile. By knowing the axial force (axial force coefficient), it is possible to predict the impact velocity of the projectile, by external ballistic calculation, in function of the distance of the target, and to define the maximum effective range from the aspect of terminal ballistics. In this paper two models will be presented for predicting axial force (the axial force coefficient) of an APFSDS projectile after discarding sabot. The first model is defined in STANAG 4655 Ed.1. This model is used to predict the axial force coefficient for all types of conventional projectiles. The second model for predicting the axial force coefficient of an APFSDS projectile, which is presented in the paper, is the CFD model (eng. Computed Fluid Dynamics).
\end{abstract}

Keywords: APFSDS, axial force coefficient, STANAG 465.5 Ed.1, CFD, PRODAS

\section{Introduction}

Ammunition based on the use of kinetic energy (KE) penetrates through targets primarily with the energy of a projectile body or sub-projectile (penetrator), which is made of high-density metal. The efficiency of this ammunition depends on the impact velocity of the projectile at the target, the length, diameter and density of the penetrator. A representative of modern kinetic energy ammunition, which is mostly used today in armies around the world, is APFSDS ammunition (Armor Piercing Fin Stabilized Discarding Sabot), which is made of an alloy of tungsten or depleted uranium [1].

The components of modern armor piercing fin stabilized discarding sabot ammunition are shown schematicallyy in Figure 1:

1. penetration with fin stabilizer,

2. sabot,

3. combustible case,

4. propellant,

5. primer assembly, and

6. case base.

This work is licensed under a Creative Commons Attribution License (https://creativecommons.org/licenses/by/4.0/ ) that allows others to share and adapt the material for any purpose (even commercially), in any medium with an acknowledgement of the work's authorship and initial publication in this journal. 


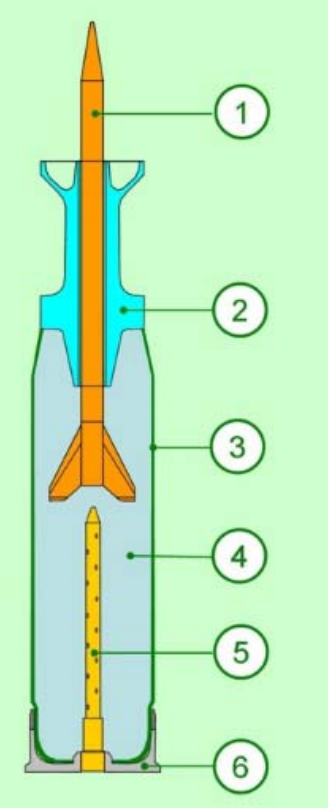

Figure 1. Main components of APFSDS ammunition [2]

During the movement of the APFSDS projectile through the barrel of the weapon, the energy of the propellant charge acts on the bottom of the projectile (the penetrator is still connected to the sabot) and drives it. After the projectile comes out of the barrel, due to the difference in resistance and mass of the sabot and penetrator, the sabot separates and the penetrator continues to fly towards the target (Figure 2). When moving through the barrel of the cannon, the projectile with the sabot reaches supersonic velocities (up to $5 \mathrm{Mach}$ ).

APFSDS projectiles are statically stabilized projectiles. Stabilization is provided using aerodynamic surfaceswings. When determining the axial force (axial force coefficient), the projectile body and the wings are observed separately. The sum of these two components of the axial forces on the APFSDS projectile gives the total axial force (axial force coefficient) [4].
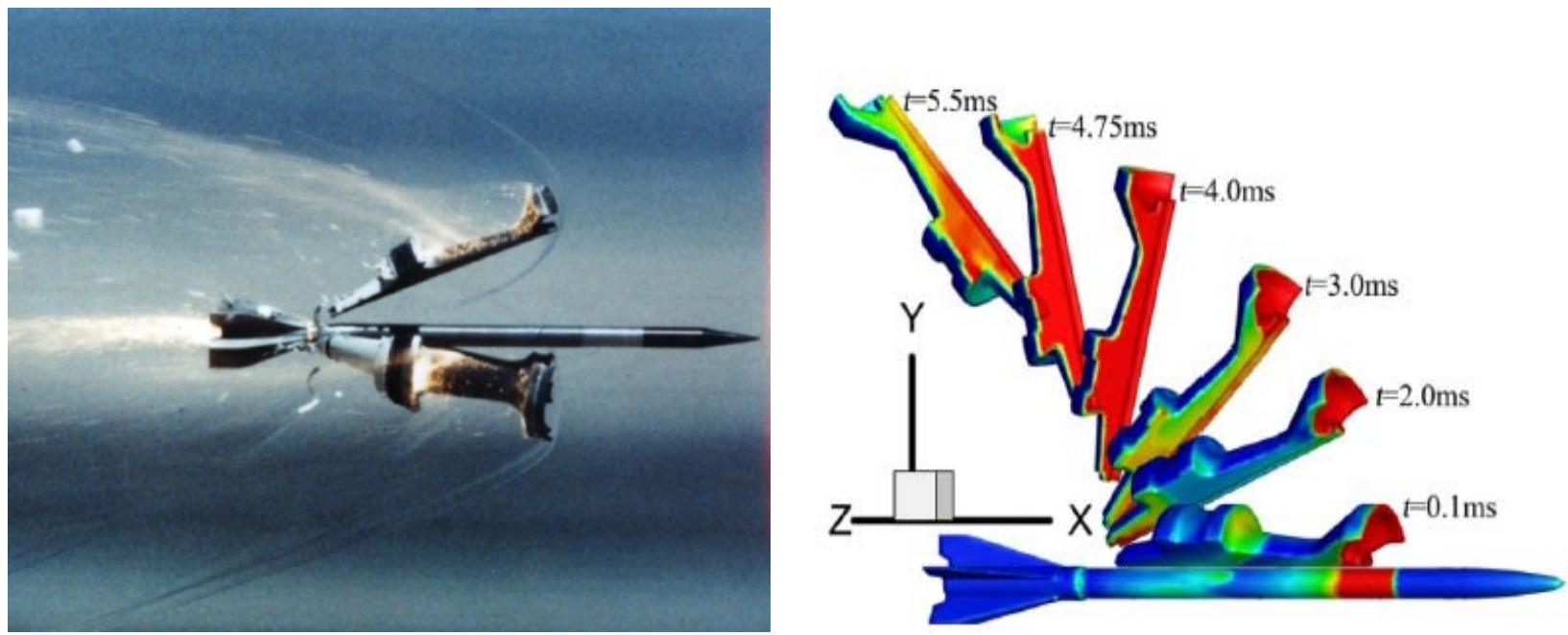

Figure 2. Process of discarding sabot from APFSDS projectile [3]

\section{Models for prediction of coefficient of axial force}

There are many methods for predicting the axial force coefficient of APFSDS projectiles, but all of them are based on the application of three general methods:

1. experimental (wind tunnel or polygon),

2. theoretical, and

3. numerical (CFD - Computational Fluid Dynamics). 
Experimental methods determine aerodynamic coefficients in an air (aero) tunnel or on the basis of measuring the movement of a projectile in flight (as a material point or a rigid body). This method gives the most realistic values for the axial force coefficient; however, the disadvantages of the tunnel experiment are [5]:

- high price,

- scaling problems if the model is not life-size,

- interference from tunnel walls,

- measurement difficulties.

Assumptions and simplifications are necessary in theoretical methods for the solving problems. This includes simplifying geometry and simplifying equations. Equations known as Navier-Stokes equations, along with the energy equation and the continuity equation, describe the flow of fluid around a body. They are analytically unsolvable in closed form, but can be simplified for specific geometry or flight conditions [5].

Numerical methods are new; they have been used since the advent of computers during World War II. Advanced CFD codes numerically solve Navier-Stokes equations and can show the complete flow field around an object for specific flight conditions. With these methods, problems arise in determining the boundary conditions, because the initial conditions must be defined with great accuracy [5].

In the continuation of this chapter, two models for predicting the axial force coefficient APFSDS projectiles will be presented. The first model is presented in the STANAG 4655 Ed.1 standard. The second model for predicting the axial force coefficient of an APFSDS projectile is the numerical model (CFD). The program to be used for the numerical simulation of projectile flow is ANSYS Fluent. The presentation of the models as well as the results of the calculations will be shown below.

\subsection{Model defined in standard STANAG 4655 (Ed.1)}

The standard, STANAG 4655, shows an engineering model for prediction of the aerodynamic coefficients of conventional projectiles. The details of the standard are given and are divided into three parts [6]:

1. Body Aerodynamics

2. Fin Aerodynamics

3. Generalized Yaw Aerodynamics

The axial force of a projectile can be divided into two parts: pressure axial force and viscous (friction) axial force. The complete axial force coefficient $C_{x}$ is finally obtained by summing up the relevant, separately calculated pressure axial force components and the viscous axial force obtained for entire wetted area. The total axial force coefficient of APFSDS projectile (without sabot) is [6]:

$$
C_{x}=C_{x_{\text {body }}}+C_{x_{\text {fin }}}
$$

where $C_{x_{\text {body }}}$ is axial force coefficient of projectile body and $C_{x_{f i n}}$ is axial force coefficient of fins.

\subsubsection{Axial force computation methods for projectile body}

The axial force of a projectile consists of the pressure axial force of the nose, base (including possible tail boom), protruding (driving band, grooves and steps), and of the viscous axial force as a sum of the following form [6]:

$$
C_{x_{\text {body }}}=C_{x_{n}}+C_{x_{b}}+C_{x_{p r}}+C_{x_{f}}
$$

where $C_{x_{n}}$ is axial force coefficient of the nose, $C_{x_{b}}$ is axial force coefficient of the base, $C_{x_{p r}}$ is axial force coefficient of the protruding and $C_{x_{f}}$ is viscous axial force coefficient.

The axial force coefficient of the nose at supersonic region for a cone is calculated according to the formula (3) giving the pressure coefficient on the nose surface [6]. The second term takes into account the nose shape on drag force (see Fig. 3).

$$
C_{x_{n}}=C_{p}=k_{1} \sin ^{2} \varepsilon+k_{2} \frac{\sin \varepsilon(\cos \varepsilon)^{M^{-R R}(16,5 R R-2,5) \sin \varepsilon}}{M+[R R(1-R R)]^{0,5} \cos \varepsilon} \quad(M \geq 1)
$$

The coefficient $k_{l}$ is an average pressure coefficient on a blunt projectile face behind a normal shock wave and the coefficient $k_{2}$ takes into account the shape of the nose as a function of radius ratio parameter $R R$ [6]. 

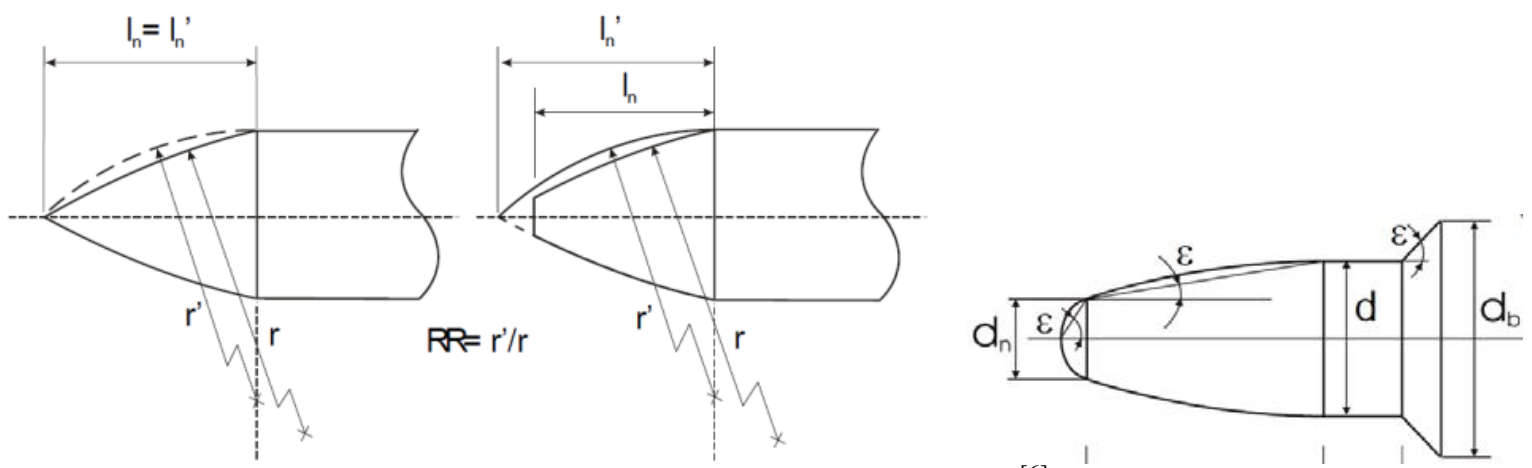

Figure 3. Variable RR (Radius Ratio) ${ }^{[6]}$

The coefficient $k_{1}$ is computed from (4), and the coefficient $k_{2}$ is computed according to (5). The radius ratio $R R$ in the formulae is an inverse of the ratio of the true radius of curvature and the tangent-ogive radius $r$ ' (formula 6). The nose contour line is to be extended to the projectile center line in case of blunted nose (see Figure 3). The extended nose length is used in formula (6). The ratio $R R$ is zero for cones [6].

$$
\begin{gathered}
k_{1}=\frac{5}{3}-\frac{2}{3 M^{\sqrt{2}}} \quad(M \geq 1) \\
k_{2}=0,9-0,9 R R+R R^{2} \\
r^{\prime}=\frac{l_{n}^{2}}{d}+\frac{d}{4}
\end{gathered}
$$

The axial force coefficient of the base $\boldsymbol{C}_{\boldsymbol{x}_{\boldsymbol{b}}}$ is computed from formula (7) [6]:

$$
C_{x_{b}}=-C_{p_{b}}\left(\frac{d_{b}}{d}\right)^{2}
$$

The pressure coefficient $C_{p_{b}}$ is computed as [6]:

$$
C_{p_{b}}=C_{p_{b_{c}}}\left(\frac{d_{b}}{d}\right)^{x} \quad x=2 \text { when } M a<0.9, \text { otherwise } 1
$$

The pressure coefficient on the base of a long cylinder $C_{p_{b_{c}}}$ is computed at supersonic speeds $(1.1<\mathrm{Ma})[6]$ :

$$
C_{p_{b_{c}}}=-0,31 e^{-0,37 M}
$$

Viscous axial force coefficient $\boldsymbol{C}_{\boldsymbol{x}_{\boldsymbol{f}}}$ is calculated by formula [6]:

$$
C_{x_{f}}=C_{f} \frac{S_{w e t t e d}}{S}
$$

where $C_{f}$ is average friction coefficient (11 or 12) for a smooth flat, $S_{\text {wetted }}$ is computed wetted surface area and $S$ is reference area $\frac{\pi d^{2}}{4}$.

The turbulent boundary-layer friction coefficient $C_{f}$ is computed by equation [6]:

$$
C_{f}=\frac{0,455}{(\log R e)^{2,58}}\left(1+0,21 M^{2}\right)^{-0,32}
$$

where $R e$ is Reynolds number $\frac{V l}{v}, l$ is projectile/nose length and $v$ is kinematic viscosity.

The laminar boundary-layer friction coefficient $C_{f}$ is computed by equation [6]:

$$
C_{f}=\frac{1,328}{\sqrt{R e}}\left(1+0,21 M^{2}\right)^{-0,12}
$$


The kinematic viscosity $v$ is computed from [6]:

$$
v=\frac{\mu}{\rho}
$$

The air density $\rho$ is computed according to ICAO standard atmosphere. The dynamic viscosity $\mu$ is obtained from the Sutherland formula [6]:

$$
\mu=\frac{C_{1} T^{1,5}}{T+C_{2}}
$$

where $C_{1}=1,458 e^{-6}\left[\frac{\mathrm{kg}}{\mathrm{ms} / \mathrm{K}}\right], C_{2}=110,4[\mathrm{~K}]$ and $T$ is air temperature, obtained from ICAO atmosphere model.

The axial force coefficient of protruding $\boldsymbol{C}_{\boldsymbol{x}_{\boldsymbol{p}}}$ is computed by estimating the forward and backward facing surface pressure drag separately [6]:

$$
C_{x_{p r}}=C_{x_{\text {groove }}}+\Delta C_{x_{g}}
$$

The pressure coefficient sum (of the backward and forward facing parts) $C_{x p_{\text {groove }}}$ will change linearly between the sum and 0 when the ratio $e / h$ (width/depth of groove) goes from 7 to 0 [6].

$$
C_{x_{\text {groove }}}=\frac{e}{7 h} C_{x p_{\text {groove }}} \quad\left(\frac{e}{h}<7\right)
$$

The formulae for the pressure coefficients at velocities above speed of sound are [6]:

$$
\begin{gathered}
C_{p_{F W}}=(-0,067(M-1)+0,4) \sin \vartheta \\
C_{p_{B W}}=-0,65 M^{-1,68}
\end{gathered}
$$

where $\vartheta$ is angle of grove profile.

Certain types of finned projectiles have a relatively large groove pattern on the surface of the cylindrical part of body. These grooves are needed at the internal ballistic phase and after launch, the grooves cause an unfavorable flow retarding effect.

The axial force coefficient of excessive amount of grooves (see Figure 4) is computed in from formula [6]:

$$
\Delta C_{x_{g}}=1,6 \frac{l_{g}}{l_{c}} C_{x_{f}}\left(C_{\text {wetted }}-1\right)
$$

The coefficient $C_{x_{f}}$ is the viscous drag coefficient (equation 10) of body cylinder part and the coefficient $C_{\text {wetted }}$ is used to take into account the groove depth on drag. The coefficient is the surface area ratio of grooved cylinder length to that of same length cylinder without grooves; the incremental drag will be zero in case the surface coefficient $C_{\text {wetted }}$ is 1 [6].

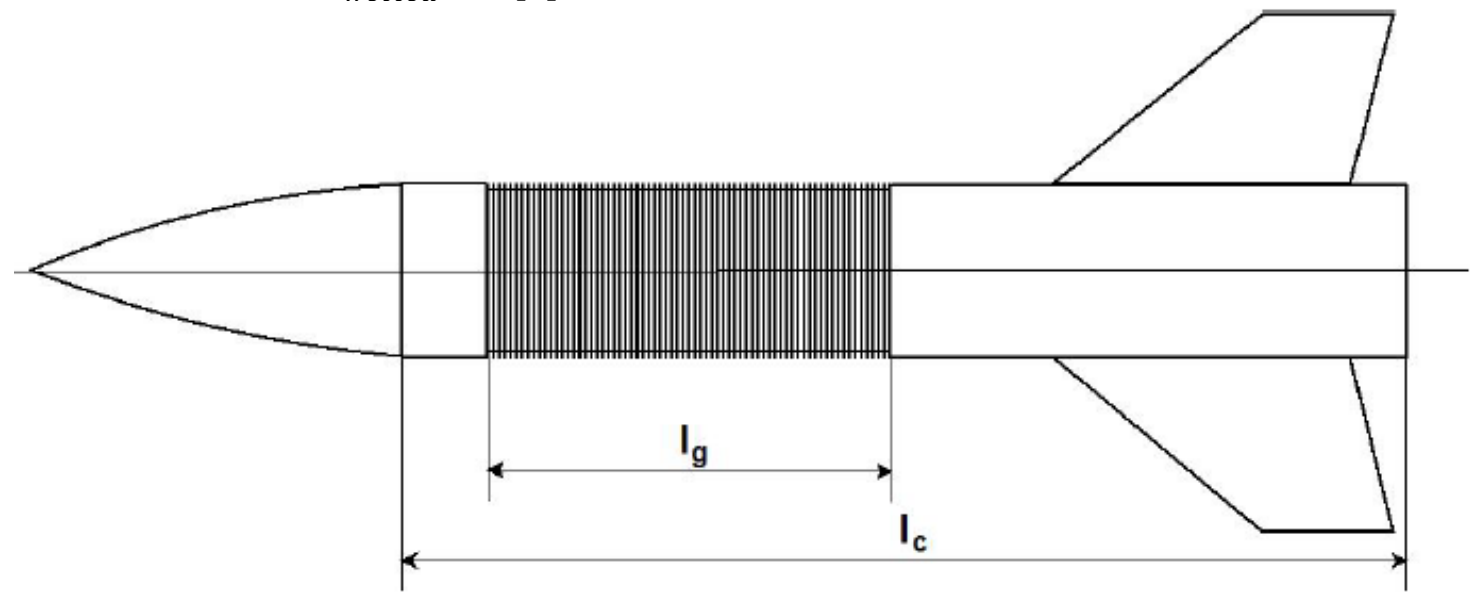

Figure 4. Groove pattern area on the surface of the cylindrical part of body [6] 


\subsubsection{Axial force computation methods for projectile fins}

The axial force coefficient of fins $\left(C_{x_{f i n}}\right)$ is computed in the function of Mach number, but also some effect of Reynolds number is introduced via skin friction. The reference area is a circle area based on the diameter of the projectile cylindrical part.

The axial force coefficient of fins consists of the wave drag $\left(C_{x_{f i n} \text { wave }}\right)$, axial force coefficient of leading edge $\left(C_{x_{f i n_{L E}}}\right)$, axial force coefficient of trailing edge $\left(C_{x_{f i n_{T E}}}\right)$ and viscous force coefficient of fins $\left(C_{x_{f i n_{\text {viscous }}}}\right)$ as a sum of the following form [6]:

$$
C_{x_{f i n}}=C_{x_{\text {fin }} \text { viscous }}+C_{x_{\text {fin wave }}}+C_{x_{f i n_{L E}}}+C_{x_{f i n_{T E}}}
$$

The wave drag at supersonic speeds for fins with sharp leading/tailing edges (see Fig. 5) is calculated according to the formula (21) [6]:

$$
C_{x_{\text {fin }} \text { wave }}=n_{\text {fin }} \frac{K}{M}\left(\frac{t}{c}\right)^{2} \frac{S_{\text {fin }}}{S}
$$

where: $K$ is shape correction factor (see Fig. 5), $\frac{t}{c}$ is average fin thickness ratio, $S$ is reference area, $S_{f i n}$ is fin area (see Fig. 6), $n_{\text {fin }}$ is number of fins.

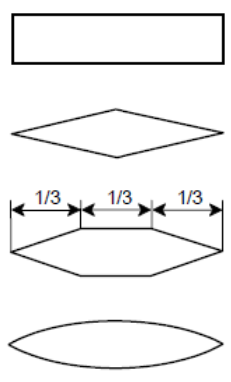

Figure 5. Airfoil shape correction factor K [6]
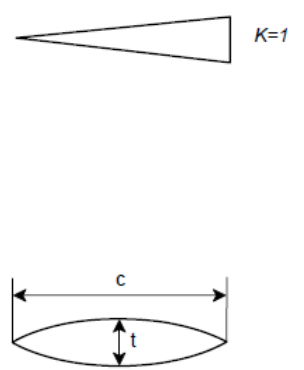

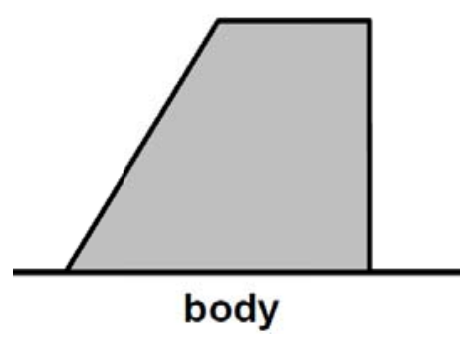

Figure 6. Projected area of fins [6]

The formula (21) is applied when $M_{L E} \geq 1$ (Mach number normal to leading edge). $M_{L E}$ is supersonic if $\mu>$ $\wedge_{L E f i n}$, where $\wedge_{L E f i}$ is sweep angle of fin leading edge (see Fig. 7). The Mach angle $\mu$ is then computed from expression [6]:

$$
\mu=\arcsin \left(\frac{1}{M}\right)
$$

The drag coefficient value is taken to be constant down to free-stream Mach number 1 if $\mu<\wedge_{\text {Lefin }}[6]$.

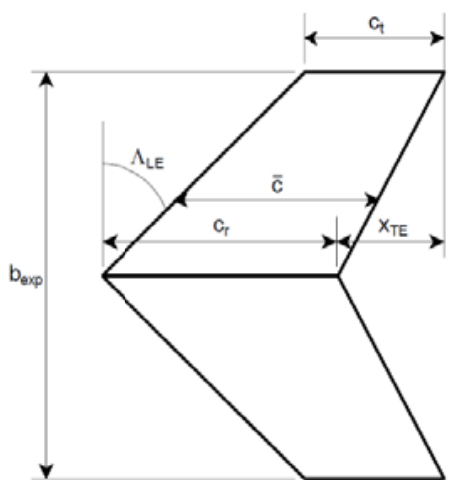

Figure 7. Exposed wing geometry without body [6]

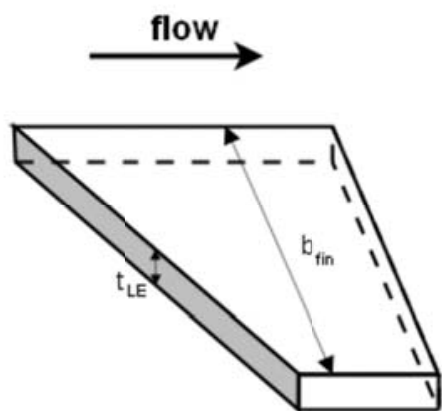

Fig. 8. Schematic of fin blunt leading edge geometry [6]

Axial force coefficient of leading edge is obtained by multiplying $C_{P}$ by the number of fins $\left(n_{\text {fin }}\right)$, taking the reference area $(S)$ and fin dimensions (Fig. 8) into account [6]: 


$$
C_{x_{f i n_{L E}}}=\frac{C_{P} t_{L E} b_{f i n}}{S} n_{f i n}
$$

The blunt leading edge average pressure coefficient $C_{P}$ is estimated by utilizing formula (24) [6]:

$$
C_{P}=\cos ^{2} \wedge_{L E_{f i n}} C_{p}
$$

Parameter $C_{p}$ is estimated by utilizing formula [6]:

$$
C_{p}=k 1
$$

Axial force coefficient of trailing edge is obtained by multiplying it by the number of fins $\left(n_{\text {fin }}\right)$, taking the reference area $(S)$ and fin dimensions (Fig. 9) into account [6]:

$$
C_{x_{f i n} T E}=\frac{C_{P} t_{T E} b_{f i n}}{S} n_{f i n}
$$

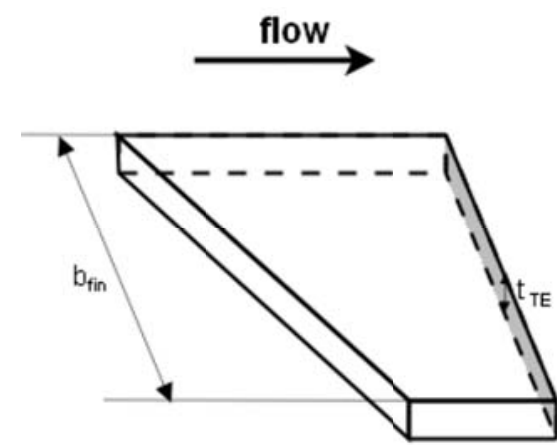

Figure 9. Schematic of fin blunt trailing edge geometry [6]

The average pressure coefficient on a fin blunt trailing edge is computed at supersonic regions according to formula [6]:

$$
C_{P}=-0,65 M^{-1,68}
$$

Viscous force coefficient of fins is calculated by same formulae (10) as in projectile skin friction drag. In fin case $S_{\text {wetted }}$ is the wetted surface area of fins, instead of the wetted surface area of projectile body and flow is consider to be turbulent [6].

$$
C_{x_{\text {fin }} \text { viscous }}=C_{f} \frac{S_{\text {wetted }}}{S}
$$

The turbulent skin friction coefficient $C_{f}$ is computed by formula [6]:

$$
C_{f}=\frac{0,455}{\left(\log R e_{\bar{c}}\right)^{2,58}}\left(1+0,21 M^{2}\right)^{-0,32}
$$

where: $R e_{\bar{c}}$ is Reynolds number $-\frac{V \bar{c}}{v}, \bar{c}$ is Mean Aerodynamic Chord (see Fig. 7), $v$ is kinematic viscosity.

\subsection{CFD-model (Computed Fluid Dynamics)}

Numerical simulation methods, using computed fluid dynamics, are an important aspect of modern research because they complement experimental and analytical models, reducing total time and labor costs. In the experimental approach, most of the time is spent designing the experiment and making the model. In the computational approach, most of the time is spent on generating a geometric mesh (if the flow area is geometrically complex) and later analyzing the results.

The main disadvantage of the computer approach is limitation to problems for which there is a reliable physical / mathematical model [7].

In the general case, a numerical computational simulation consists of several main steps (Fig. 10):

- Problem identification involves defining the objectives of numerical simulation, what are the modeling options, which physical models will be included in the analysis (viscosity, turbulence, 
compressibility), what simplifications can be used, whether user-defined functions should be used, what accuracy is required and how long it takes to get results [8].

- Preprocessing involves defining the geometry, mesh (space discretization), physical model, and solver used [8].

- Solver settings include solver type selection, discretization scheme, solution initialization, convergence monitoring (stability analysis), and accuracy check (mesh independence check and mesh adjustment on a specific part of the domain) [8].

- In the post-processing process, the results are examined and analyzed to understand the solution and extract useful data. Visualization tools in numerical programs make it possible to gain insight into the field of pressures or velocities, to visualize flow vectors, to predict the position of shock waves. [8].

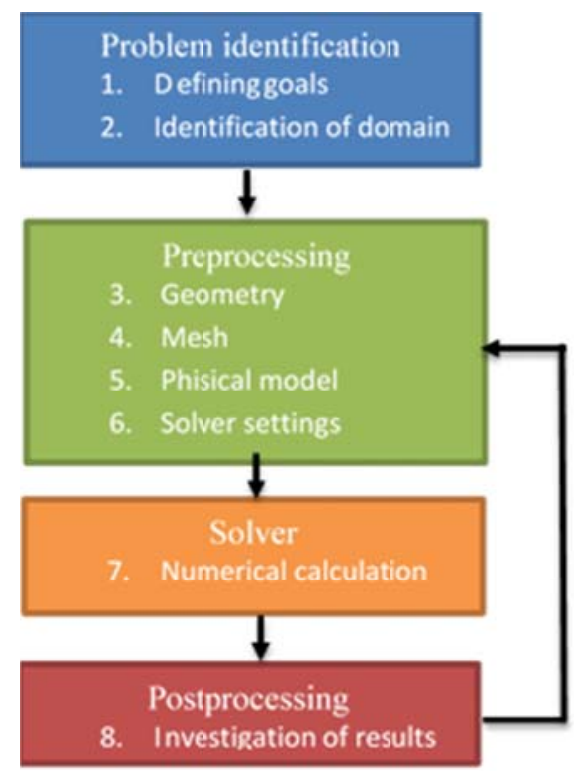

Figure 20. Process of numerical simulation [8]

\subsubsection{Mathematical model}

Each simulation is based on a mathematical model, which denotes the mathematical notation of a physical model. The mathematical model includes the following assumptions [7]:

- Air is a continuum.

- Air is considered a homogeneous mixture of gases.

- The physical properties of air are the same in all directions - the air is isotropic.

- Air is a single-phase fluid.

- Mass forces are neglected.

Continuity behavior can be described by transport equations based on the basic laws of mass conservation, momentum, and energy. The equations derived from the given laws are presented in integral form for an arbitrarily selected part of the continuum, the volume $\Omega$ bounded by a closed area - the limit of the control volume $d \Omega$ (Figure 11). The surface element $d S$ is defined by the unit vector of the normal $\vec{n}$ [7].

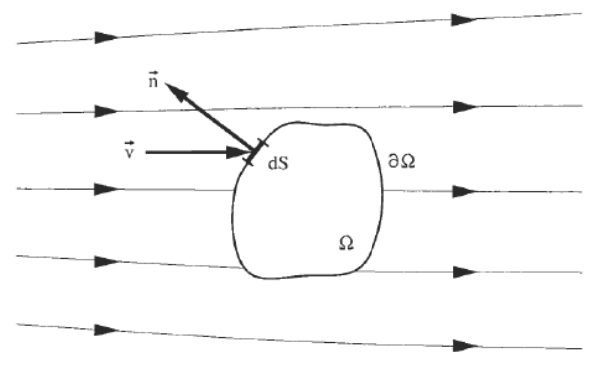

Figure 11. Control volume [7] 
The characteristics of the air flow (pressure, tangential stress, velocity, temperature, etc.) are determined, at each point in space and at any time, by a system of differential equations [9]:

- Law of mass conservation:

$$
\frac{\partial}{\partial t} \int_{\Omega} \rho d \Omega+\oint_{\partial \Omega} \rho(\vec{v} \cdot \vec{n}) d S=0
$$

- Law of momentum conservation:

$$
\frac{\partial}{\partial t} \int_{\Omega} \rho \vec{v} d \Omega+\oint_{\partial \Omega} \rho \vec{v}(\vec{v} \cdot \vec{n}) d S=-\oint_{\partial \Omega} p \vec{n} d S+\oint_{\partial \Omega}(\overline{\bar{\tau}} \cdot \vec{n}) d S
$$

- Law of energy conservation:

$$
\frac{\partial}{\partial t} \int_{\Omega} \rho E d \Omega+\oint_{\partial \Omega} \rho E(\vec{v} \cdot \vec{n}) d S=\int_{\Omega} k(\nabla T \cdot \vec{n}) d \Omega-\oint_{\partial \Omega} p(\vec{v} \cdot \vec{n}) d S+\oint_{\partial \Omega}(\overline{\bar{\tau}} \cdot \vec{v}) \vec{n} d S
$$

where $\vec{v}$ is velocity of airflow, $p$ is pressure, $\rho$ is density, $T$ is temperature, $E$ is total energy, $\overline{\bar{\tau}}$ is stress tensor.

\subsubsection{Simulation of air flow around projectile}

The following will be adopted for all simulations [9]:

- The problem will be observed as 3D geometry problem (because of fins).

- The working fluid is air, an ideal gas, which is modified in accordance with compressibility and changes in thermo-physical characteristics with the temperature. Density and viscosity depends on temperature, where $c_{p}$ and thermal conductivity are considered constant.

- The flow around the projectile is compressible and turbulent ( $k-\varepsilon$ model of turbulence was used).

- Spatial domain discretization will be with a hybrid mesh.

- The numerical density-based solver will be used, which simultaneously solves the equations of continuity, amount of momentum and energy. This method was developed for compressible highspeed flows.

- The equations will be linearized in implicit form, i.e. for given variables; unknown values in each cell will be computed using relations that include both existing and unknown values from adjacent cells.

- A uniform air flow encounters a projectile at a zero yaw angle.

In order to reduce the number of finite elements, only the projectile segment was taken - at an angle of $60^{\circ}$ (Figure 12). The calculation domain is limited by the external boundary of the projectile, symmetry planes and the outer boundary set at distances of 3 projectile lengths from the shell, 7 projectile lengths from the bottom and 6 lengths from the top (cylinder-shaped mesh) to avoid disturbances in free stream [9].
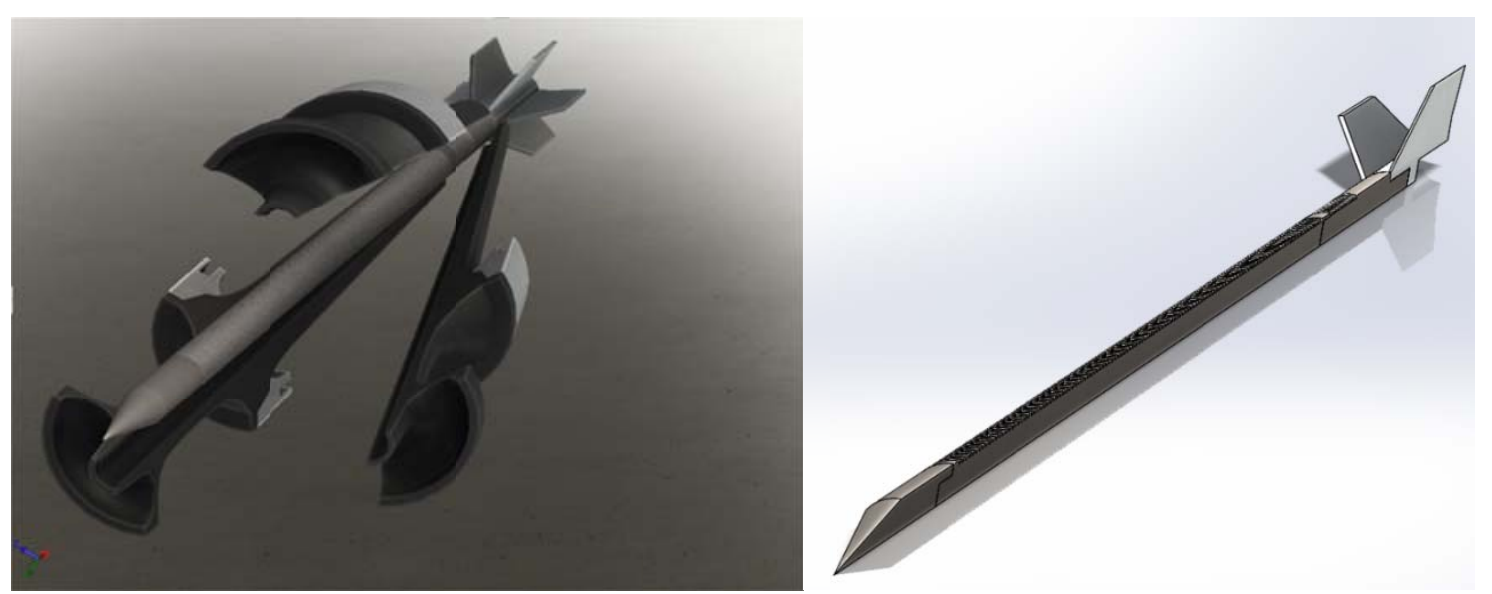

Figure 12. 3D model of APFSDS projectile $120 \mathrm{~mm}$ M829A2 [9] 
The following types of boundary conditions were selected (Figure13) [9]:

- The "wall" boundary condition, which is used to separate the regions of fluid and solid matter, is placed on the outer boundary of the projectile. At the "wall" boundary condition, the "stationary wall" and "no-slip" options were chosen, because in the case under consideration, viscous effects cannot be ignored.

The mass flux through the "wall" boundary is zero, and the pressure values at this boundary are obtained by extrapolation from inside the solution domain.

- The "symmetry" boundary condition was used as a plane of axisymmetric geometry.

- The "pressure far field" boundary condition, which is used to model the parameters of the compressible free stream at infinity, is set at the outer boundary of the calculation domain for given problem.

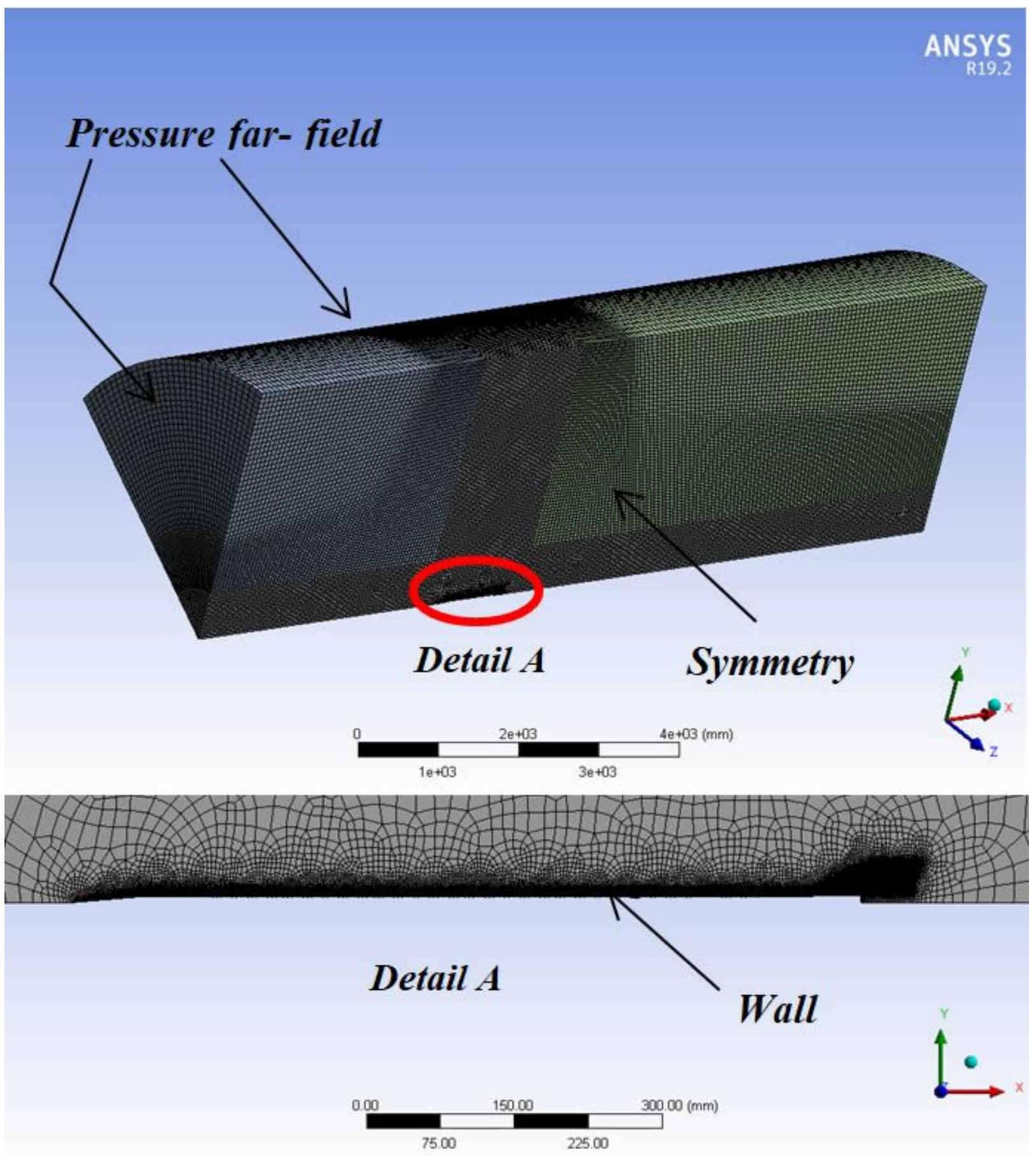

Figure 13. Generated mesh around projectile $120 \mathrm{~mm}, \mathrm{M} 829 \mathrm{~A} 2$, and boundary conditions applied [9] 


\section{Results and discussion}

For prediction of the accuracy of the engineering model (from the NATO related STANAG 4655 standard) and CFD numerical model, a comparison was made in the research with the PRODAS model. For calculation of axial force coefficients, APFSDS projectile, $120 \mathrm{~mm}$, M829A2, was chosen. The reason for choosing this projectile model is because the aerodynamic coefficients are available from PRODAS program for this projectile model.

The PRODAS software was developed to satisfy a need for rapid performance evaluation of ammunition characteristics. The development of an effective design/analysis tool for use by the design engineer in the development and evaluation of projectiles has been a multi-year project which began at General Electric in 1972 and has continued at Arrow Tech Associates, Inc. since 1991. The developed tool is now called PRODAS which is an acronym for the Projectile Design/Analysis System [10].

From the smallest match bullets, to GPS guided artillery shells, PRODAS brings together:

- Modeling - Building a model from a drawing or even a picture.

- Aerodynamics - Comparing aerodynamic coefficients from multiple aero estimators.

- Launch Dynamics - Interior ballistics, balloting and jump.

- Trajectories - Fly 4DOF, 6DOF and Body Fixed and Guided Trajectories.

- Terminal Effects - Estimate penetration of KE projectiles and lethality of fragmenting or shaped charge warheads.

- System Effectiveness - Using focused analysis or general purpose macros, compare projectiles or even GN\&C algorithms [10].

\subsection{STANAG 4655 vs. PRODAS}

The axial force coefficients of the projectile body, predicted using the model from STANAG 4655 and PRODAS models are shown in Figure 14. The axial force coefficients of the projectile body predicted by the model from STANAG (Figure 14) shows a significant difference in the range of Mach 3 to 5. It can be seen that this difference decreases with increasing Mach number [9].

The axial force coefficients of the projectile fins, predicted using the model from STANAG 4655 and PRODAS models are shown in Figure 15. The downward trend in the value of the axial force coefficient of the fins in the STANAG model is higher than in the PRODAS model. The differences between the values decrease slightly with increasing Mach number (Figure 15) [9].

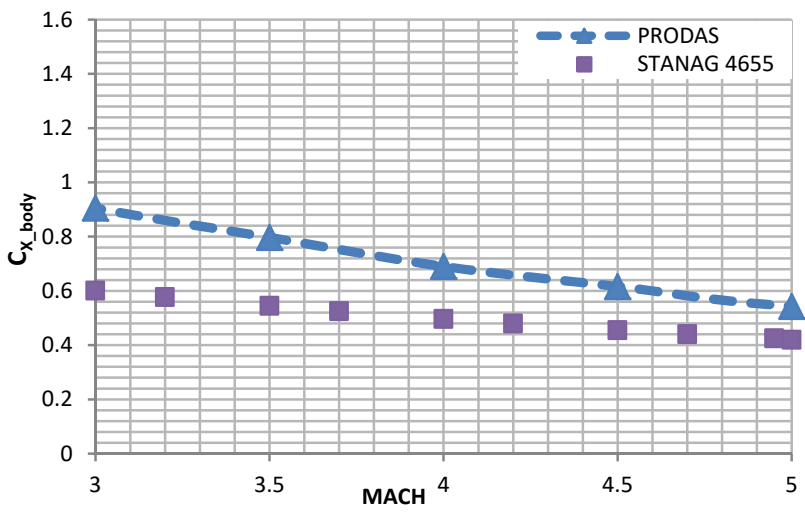

Figure 14. Coefficients of axial force of the projectile body (120 mm, M829A2) [9]

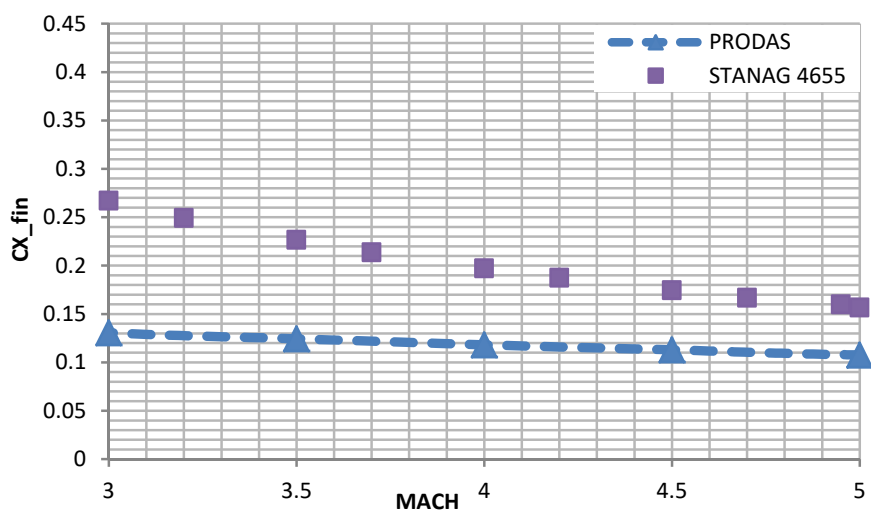

Figure 15. Coefficients of axial force of the projectile fins (120 mm, M829A2)

The axial force coefficients of the projectile obtained by the model from the STANAG 4655 standard, are smaller than the coefficients obtained by the PRODAS model (for projectile $120 \mathrm{~mm}$, M829A2). The difference between the values of the coefficients obtained with model from STANAG and PRODAS, decreases with increasing Mach number (Figure 16).

The percentage difference of the coefficients obtained by the model from the STANAG in relation to the coefficients obtained by applying the PRODAS model is given in figure 17.

From figure 17 it can be noticed that the largest percentage difference between the predicted values of the model from the STANAG and PRODAS is $16.3 \%$ in the range of 3 to 5 Mach [9]. 


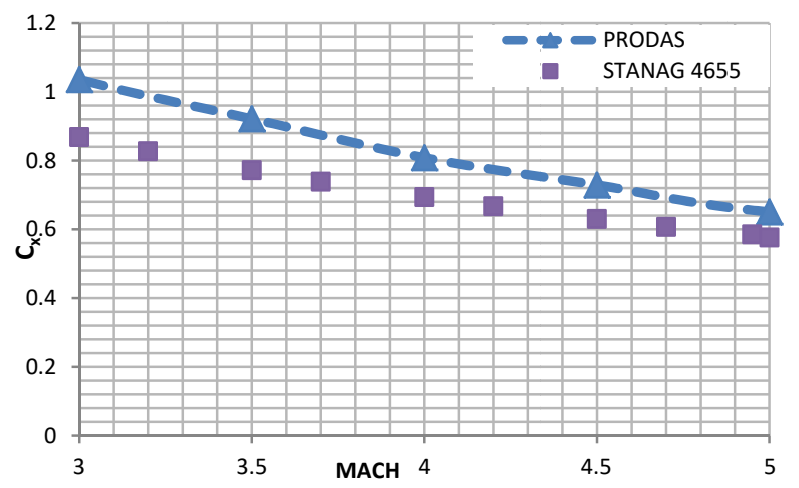

Figure 16. Coefficients of axial force of the projectile (Projectile $120 \mathrm{~mm}$, M829A2) [9]

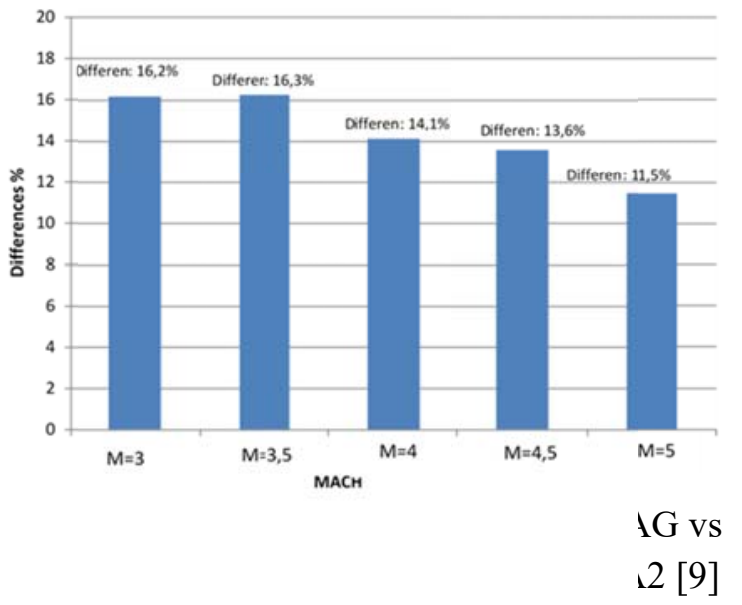

\subsection{CFD vs. PRODAS}

The results of the numerical simulation of air flow around projectile $(120 \mathrm{~mm}, \mathrm{M} 829 \mathrm{~A} 2)$, in parallel with the results of PRODAS (function $C_{x}=f(M)$ ) are shown in figure 18. The largest deviations are recorded for $\mathrm{M}=$ 3 , where the difference is about $8.4 \%$. As the Mach number increases, the difference decreases by almost 1 $\%$. The percentage difference of the coefficients obtained by the numerical simulation in relation to the coefficients obtained by applying the PRODAS model is given in figure 19 [9].

Based on the obtained results of numerical simulations, the analysis of the pressure and velocity field around the projectile $120 \mathrm{~mm}, \mathrm{M} 829 \mathrm{~A} 2$ was done. The resistance force of a fluid that opposes the motion of a body in it arises as a result of the action of a normal force and a tangential force on the surface of a body moving through the atmosphere. The source of fluid resistance to body motion are practically three natural phenomena: fluid viscosity, shock waves (at velocities $M \geq 1$ ), and turbulence flow behind the body [9].

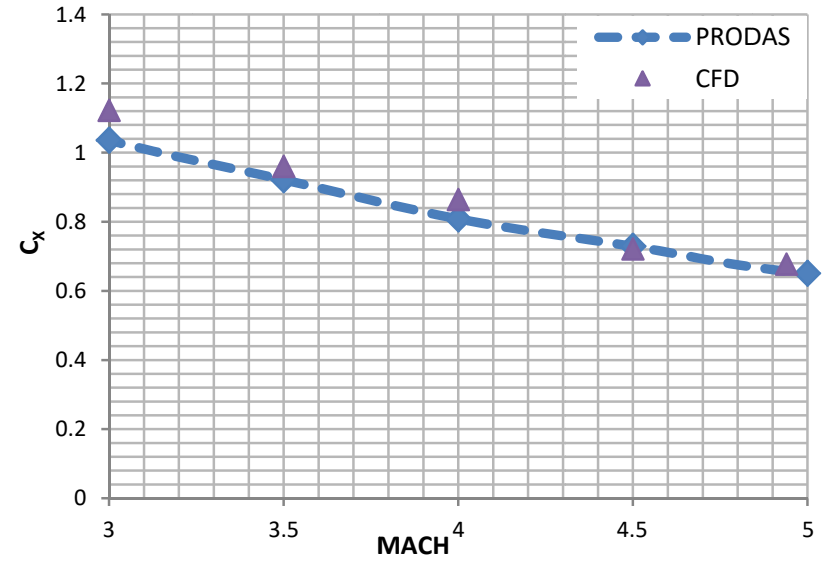

Figure 18. Coefficients of axial force of the projectile (Projectile $120 \mathrm{~mm}, \mathrm{M} 829 \mathrm{~A} 2$ ) [9]

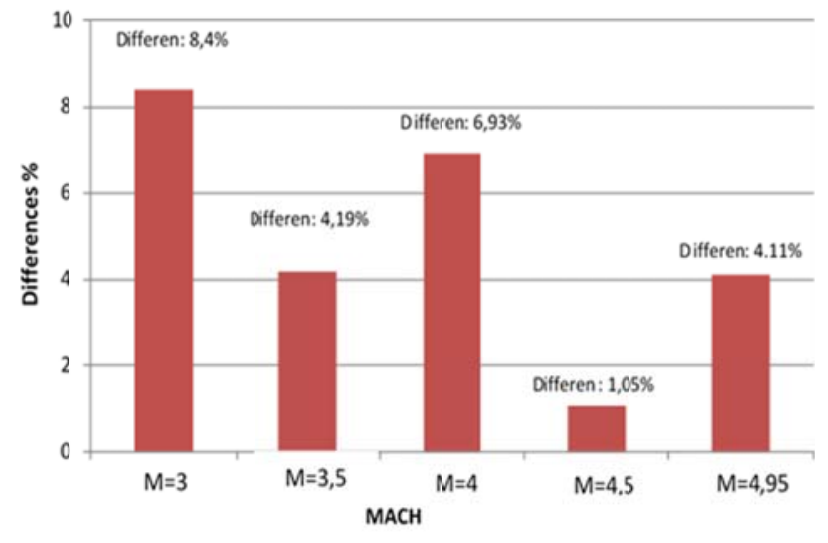

Figure 19. Relative differences (CFD vs PRODAS) Projectile $120 \mathrm{~mm}$, M829A2 [9]

The pressure and velocity fields, shown in Figures 20 and 21, are complex. From Figure 20, it can be seen that the free stream pressure is not the same for different Mach numbers. As expected for the supersonic flow, oblique shock waves appear at the top of the projectile, while a characteristic underpressure zone appears behind the projectile.

During supersonic flow, underpressure is created behind the rear part of the projectile. This underpressure causes the streams to bend towards the projectile axis. Near the axis, the streams must bend again. In supersonic flow, the bent extension of the boundary layer draws air from the rear of the projectile so that a circular movement of air is created. Generally speaking, the supersonic flow regime of any body is characterized by pronounced shock waves, extremely narrow areas of fluid in which the flow properties change discontinuously (extremely) in a very short time interval, with pressure gradients being extremely large. In front of the shock wave, there is a zone of undisturbed flow, while behind it there is a zone in which 
there are differences in the values of pressure, speed, temperature and density. Figure 21 shows the boundary layer that forms around the projectile in flight. It can also be seen that the angle of the oblique shock wave decreases with increasing velocity at which the flow is simulated [9].

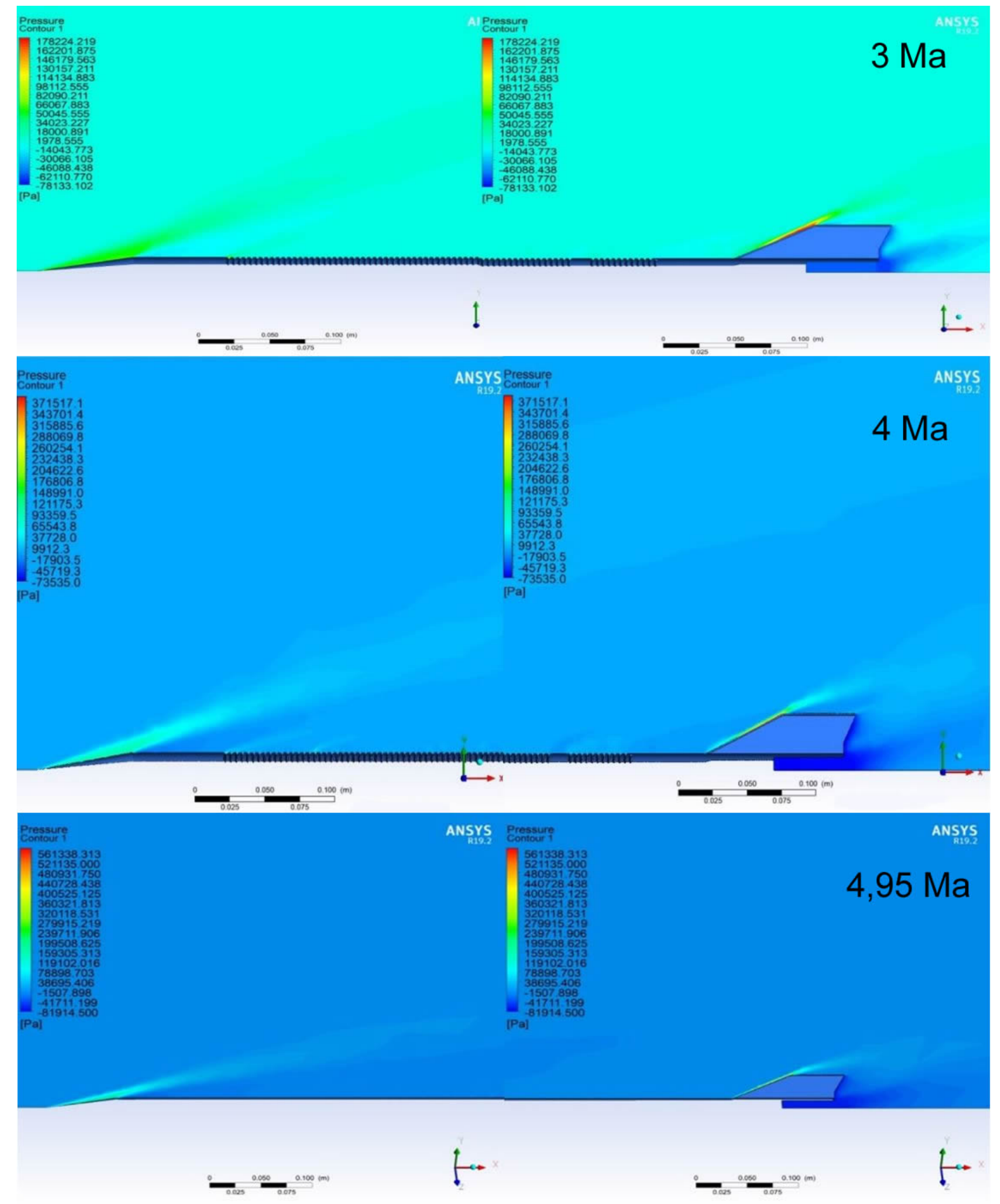

Figure 30. Pressure field around the projectile for different Mach numbers [9] 


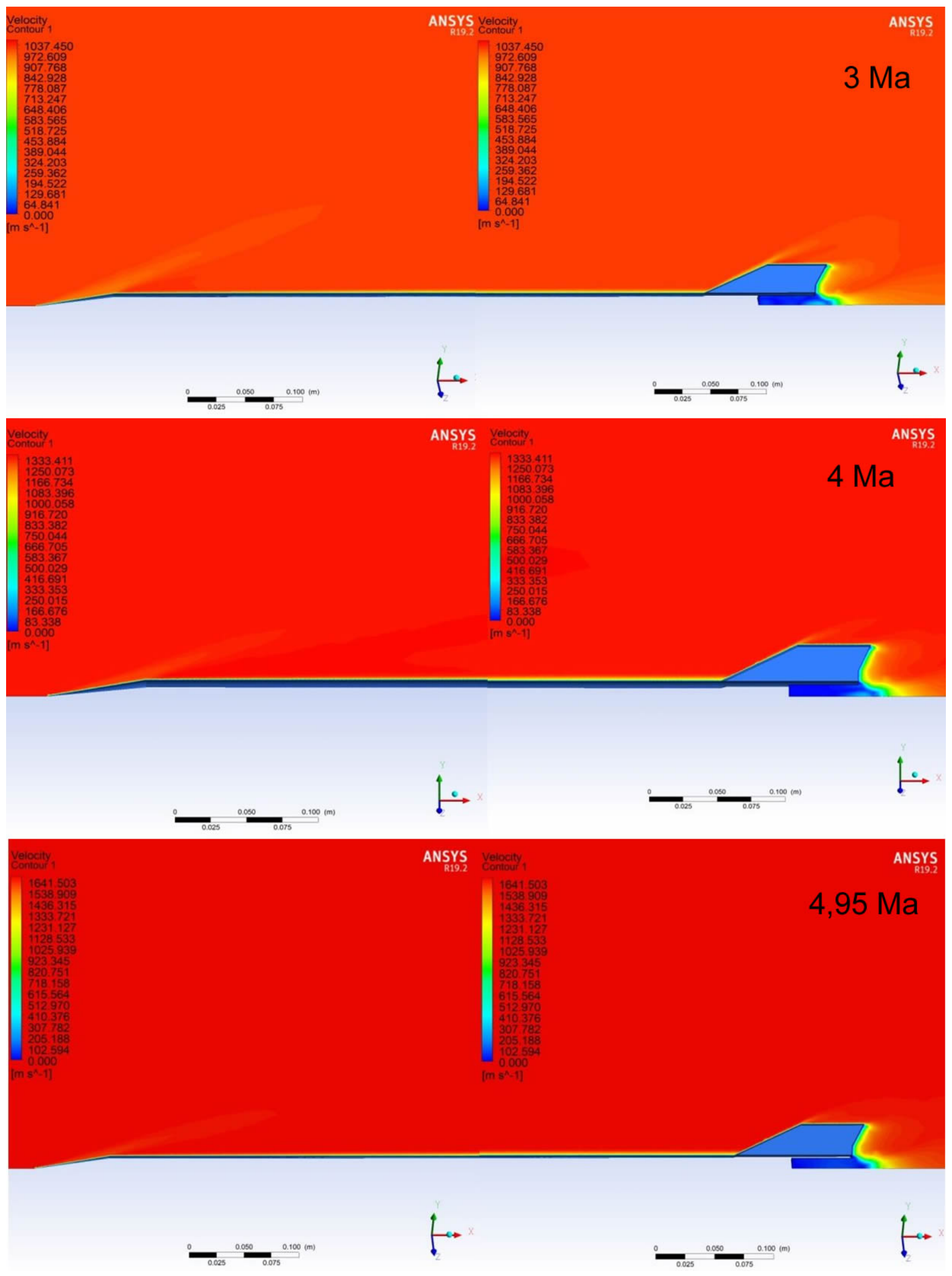

Figure 21. Field of velocities around the projectile $120 \mathrm{~mm}$, M829A2 for different Mach numbers [9] 


\section{Conclusion}

Based on theoretical considerations and analysis of available models (STANAG 4655 and CFD) for predicting the aerodynamic coefficient of axial force for wing-stabilized projectiles, the prediction of the axial force coefficient for APFSDS projectile $120 \mathrm{~mm}$, M829A2 was performed. The data obtained using the engineering model (from the STANAG 4655 standard), and the data obtained by numerical simulation of projectile flow with the available data from the PRODAS database were compared. The following was stated:

- The total axial force coefficients of the APFSDS projectile (provided with the model from the STANAG 4655 standard) have a satisfactory agreement with the total coefficient from the PRODAS model. The largest difference between the values is about $16.3 \%$. As the Mach number increases, the difference decreases.

- The advantage of the STANAG 4655 model is that it allows the calculation of coefficients based on the geometric characteristics of the projectile without the use of computers.

- The CFD model gives very good results, the values of the axial force or the axial force coefficient. Good agreement between the results of the CFD model and PRODAS indicates that the initial and boundary conditions are well set.

- The accuracy of the CFD model depends on the mesh, initial and boundary conditions. The accuracy of the CFD model can be increased by modifying the mesh (i.e. by increasing the number of finite elements).

\section{References}

[1] A. Ćatović: Anti-tank projectiles, Manual for students, University of Sarajevo, Mechanical engineering faculty, Defense Technologies Department, Sarajevo, 2019.

[2] W. Odermatt: http://longrods.ch/compo.php, October 2020

[3] Z. Huang, Z. Chen: Numerical investigation of the tree-dimensional dynamic process of sabot discard, Journal of Mechanical Science and Technology, Vol 28, No 7, 2637-2649, 2014.

[4] B. Zečević: Anti-tank Ammunition, Lectures for students, University of Sarajevo, Mechanical engineering faculty, Defense Technologies Department, Sarajevo, 2018.

[5] S. S. Kadić: Prediction of drag force at zero yaw angle for conventional artillery projectiles, Master thesis, University of Sarajevo, Mechanical engineering faculty, Defense Technologies Department, July 2007.

[6] STANAG 4655 Ed.1: An engineering model to estimate aerodynamic coefficients, NATO Standardization Agency, 18 January, 2010.

[7] S. S. Kadić: Aaerodynamic, interior and exterior ballistic request, optimization in base bleed projectile design, $\mathrm{PhD}$ thesis, University of Sarajevo, Mechanical engineering faculty, Defense Technologies Department, Jul 2014.

[8] A. Ćatović.: Prediction of terminal-ballistic parameters for natural fragmenting high-explosive projectiles using experimental data and numerical methods, $\mathrm{PhD}$ thesis, University of Sarajevo, Mechanical engineering faculty, Defense Technologies Department, Sarajevo, 2019.

[9] Blazek, J.: Computational fluid dynamics: principles and applications, Elsevier Science Ltd, Oxford, United Kingdom, 2001.

[10] A. Trakić: Axial force coefficients of APFSDS projectiles, Master thesis, University of Sarajevo, Mechanical engineering faculty, Defense Technologies Department, July 2020.

[11] PRODAS V3, http://www.prodas.com/XQ/ASP/P.400/QX/webPageXML4.htm, Arrow Tech, 2020. 